\title{
Is there a threshold for mental rotation?
}

\author{
JOSEPH S. ROSSI and CHARLES E. COLLYER \\ University of Rhode Island, Kingston, Rhode Island
}

\begin{abstract}
A threshold-like nonlinearity was found in a mental rotation experiment designed for relatively heavy sampling of small angular disparities. The value of the threshold in this experiment appeared to be around $15^{\circ}$. Results of a control experiment were that subjects easily discriminated orientations differing by as little as $6^{\circ}$ using the same stimulus materials. A threshold hypothesis represents one way to think about the flexibility and limitations of mental rotation performance.
\end{abstract}

In the mental rotation task, observers are shown a standard figure and a comparison figure that may differ from each other in angular orientation. For several types of figures, the time required to recognize that the two figures represent the same shape (rather than mirror-image shapes) is, to a close approximation, a linear function of the angular disparity between the orientation of standard figure and that of the comparison figure (Shepard \& Metzler, 1971). This linearity suggested to Shepard and his colleagues that in comparing the shapes of two similar objects differing in orientation, an observer passes through a series of internal states bearing a one-to-one relation to the physical states that an object would pass through if it were rotated from one orientation to the other (Cooper \& Shepard, 1978)-hence, the term "mental rotation." Although they have not claimed that the internal process is strictly continuous, these researchers address mental rotation as an analogue of the corresponding physical process.

The increase in response time, as a function of angular disparity, is a "large" effect. We were initially led to study mental rotation by the possibility that published demonstrations of this large effect might conceal a smaller effect: a portion of the mental rotation function extending from $0^{\circ}$ up to some limit no greater than about $30^{\circ}$, within which response time is independent of angular disparity. This possibility derived from the idea of a threshold for mental rotation. Under a threshold hypothesis, mental rotation would be unnecessary for sufficiently small angular disparities; thus, the dependence of response time on angular disparity would apply only to suprathreshold disparities. Thus, there would be a "small" nonlinearity in the overall function, attributable to the threshold.

The angular disparities used in most experiments on mental rotation include $0^{\circ}$ and $180^{\circ}$, and a few intermediate values spaced at equal intervals of $20^{\circ}$ or $30^{\circ}$. We,

These experiments represent parts of a master's thesis by the first author. Portions of Experiment 1 were presented at the 51st annual meeting of the Eastern Psychological Association, Hartford, CT, April, 1980. Preparation of this article was supported in part by National Cancer Institute Grant No. CA27821. Reprints may be obtained from J. S. Rossi, Department of Psychology, Chafee Social Science Center, University of Rhode Island, Kingston, RI 02881. however, sampled small disparity values, from $0^{\circ}$ to $30^{\circ}$, at intervals of only $3^{\circ}$.

\section{EXPERIMENT 1 \\ A Threshold-Like Nonlinearity?}

The main prediction of a threshold hypothesis is that the mental rotation response-time function should be nonlinear. This is not to deny the large effect of approximate linearity. A threshold would modify this familiar description only slightly, by introducing a zero-slope step into the function in the region from $0^{\circ}$ up the threshold itself.

\section{Method}

Subjects. Subjects were 20 graduate and senior undergraduate students (9 men and 11 women) at the University of Rhode Island. They ranged in age from 20 to 37 years (median $=26$ years), and had normal or corrected-to-normal visual acuity. Five additional subjects' data were discarded for error rates in excess of $15 \%$.

Apparatus. Apparatus consisted of two Kodak Ektagraphic randomaccess slide projectors, a Lafayette clock/counter (Model 54417), and a Standard Instrument Corporation Photo Head photocell (Model PC-503A-2).

Materials and Procedure. The stimuli presented to the observers were projections of two perspective line drawings of three-dimensional block figures similar to those used by Shepard and Metzler (1971). Each trial consisted of a simultaneous presentation of a standard and a comparison figure. Comparison figures were shown at one of 16 possible angular orientations with respect to standard orientation: $0^{\circ}, 3^{\circ}, 6^{\circ}, 9^{\circ}$, $12^{\circ}, 15^{\circ}, 18^{\circ}, 21^{\circ}, 24^{\circ}, 27^{\circ}, 30^{\circ}, 60^{\circ}, 90^{\circ}, 120^{\circ}, 150^{\circ}$, or $180^{\circ}$ of relative rotation in the picture plane.

On half the trials, the comparison figure was a rotated version of the standard, and on the remaining trials, the comparison was both reflected and rotated with respect to the standard. Both standard figures were paired with rotated and reflected comparison figures for all 16 angular disparities for a total of 64 trials. In addition, 16 practice trials were run in order to familiarize the subjects with the task. Stimuli were projected on a blank white wall in a darkened room, with the standard figure always to the left of the comparison. Subjects were seated $193 \mathrm{~cm}$ from the projection plane, and the total projected array subtended a visual angle of about $25^{\circ}$.

Subjects were instructed to respond "same" when the comparison figure was a rotated version of the standard, and to respond "different" when the comparison was a reflected version of the standard. Responses were made by pushing a switch forward for a "same" and backward for a "different" decision. Slide presentation activated a photocell that initiated a timer, stopped by the observer's response. Observers were instructed to respond as quickly as possible without guessing. Trials on which observers responded incorrectly were rerun at the end of the test session. Because of the speeded nature of the task, response 
times over $10 \mathrm{sec}$ were counted as errors. Data from observers with error rates over $15 \%$ were discarded from the analysis.

\section{Results}

The mean response times for correct "same" responses at each angular disparity are shown in Figure 1.

It is apparent from Figure 1 that the large effect of angular disparity on mental rotation response times was replicated. The linear trend was significant $[F(1,285)=$ 792.6, $p<.001]$ and large $\left(\omega^{2}=.740\right)$. The quadratic trend component was also significant $[F(1,285)=6.93$, $p<.01$ ], but very small $\left(\omega^{2}=.006\right)$. Higher order trends, combined, were not statistically significant $[F(13,285)=$ 0.93].

\section{Discussion}

With the hypothesis of a threshold in mind, a visual inspection of Figure 1 suggests that the mental rotation response-time function is not a simple straight line. Response time seems to be independent of angle from $0^{\circ}$ to about $15^{\circ}$; this local independence would be expected if there were a threshold for mental rotation of about $15^{\circ}$. Plausible statistical confirmation is provided by the significant quadratic trend in the data, although it is not certain from the statistical presence of this trend that it should be attributed to a threshold.

Most previous studies of mental rotation have not had the opportunity to test for a small nonlinearity near the $0^{\circ}$ end of the scale. (Indeed, the hypothesis of a threshold for mental rotation may be the only motivation that would encourage the design of such a test.) Although a threshold should be reflected in the type of nonlinearity that seems to be present in Figure 1, there are several considerations that work against easy measurement of the putative threshold.

First, by its very nature, the threshold nonlinearity must be a small effect. There is a large effect in mental rotation data (the linear trend component), which accounts for a good deal of the variance, and there is inevitably a lot of error variance in response-time data. This means that there is not very much variance available to be associated with a threshold nonlinearity, even in a design similar to that of Experiment 1. Second, most empirical tests for the existence of a threshold resemble attempts to prove the null hypothesis. That is, since a threshold is a level of the manipulated variable below which that variable is expected to have no effect, a demonstration of the threshold amounts to a failure to reject the null hypothesis. There are well-known logical and statistical problems with such demonstrations. Third, if the threshold is a valid construct, it is a construct applicable to individual subjects (each of whom has a threshold for mental rotation), rather than to groups. But threshold and nonthreshold models cannot be fairly compared using fits to individual subject data because they differ in strength. Also such models made predictions that differ only with respect to the small effect of the threshold nonlinearity, which is "swamped" by the noisiness of individual-level data (Collyer, 1985).

For these reasons, Figure 1 should be interpreted cautiously. The effect, if it is there, is small. (It is interesting to speculate on whether one's visual confirmation of the threshold hypothesis could include a projective judgment that would not have arisen in the absence of the hypothesis.) Further, the subthreshold region is one where the independent variable seems to have no effect on the dependent variable. Would this independence hold up under more powerful testing? Finally, the effect, if it is there, represents the aggregate of 20 subjects' performances. While the greater power of group data may be needed to discern the presence of a threshold nonlinearity, the form of the group function, in general, would not be the form of the underlying individual functions.

Having noted these reservations, let us take the significant quadratic trend and the threshold-like appearance of Figure 1 at face value. The concept of a threshold for mental rotation is not the only possible interpretation of this nonlinearity. There are at least two others. First, human observers may not be able to tell the difference between the standard and comparison figures when they are separated by a small difference in angular orientation. In this case, the $15^{\circ}$ value would represent a limit on the subject's ability to discriminate angles, rather than a measure of the subject's ability to tolerate angular disparities without normalizing the orientation of the comparison figure. Experiment 2 attempted to measure subjects' abilities to discriminate these small angular disparities. Second, the odd behavior of the data points between $0^{\circ}$ and $30^{\circ}$ might be traceable to the heavy sampling of angles within this range in Experiment 1 . The effect of the distribution of stimulus disparities on the mental rotation function is being studied further.

\section{EXPERIMENT 2}

\section{Can Observers Discriminate Between Standard and Comparison Orientations at the Threshold?}

If the subjects in Experiment 1 were unable to discriminate angular disparities of less than $15^{\circ}$, then the threshold should be characterized not as an ability (a mar-

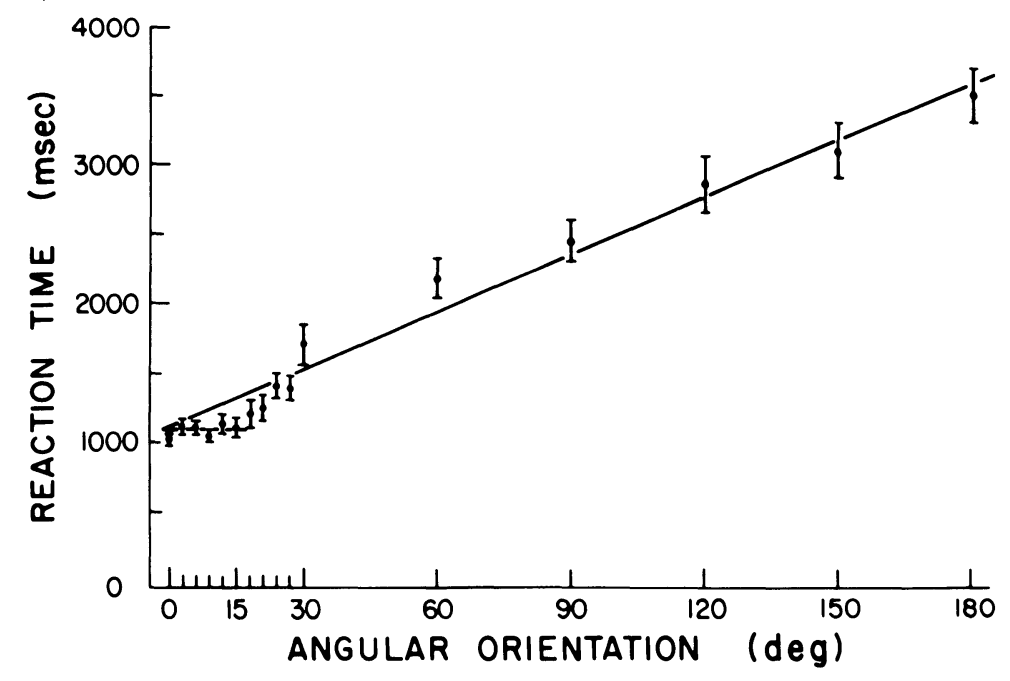

Figure 1. Mean correct "same" response times, Experiment 1. 
gin of tolerance in this type of pattern matching) but as a limitation of human information processing.

Research on figural aftereffects and on angle perception has shown that angular disparities smaller than $1^{\circ}$ or $2^{\circ}$ are difficult for observers to discriminate (Coltheart, 1971). This range is comfortably lower than our rough estimate of $15^{\circ}$ for the threshold for mental rotation. However, the aftereffect and discrimination studies have mostly used simple-line stimuli subtending small visual angles; it is not clear that a comparable level of discriminability obtains with larger and more complex figures such as the three-dimensional block projections of Experiment 1 . Furthermore, the fact that the mental rotation task requires many successive eye fixations (Just \& Carpenter, 1976) suggests a "noisy" cognitive state in which angular disparities greater than $1^{\circ}$ or $2^{\circ}$ might escape the observer's notice. Finally, observers in Experiment 1 performed the mental rotation task under time pressure. It may be that $1 \mathrm{sec}$ or so is not enough time to perceptually discriminate small angular disparities in the orientations of complex figures.

For these reasons, it seems possible that the apparent threshold for mental rotation is actually a measure of observers' inabilities to discriminate small angular disparities. While introspective judgments of the stimuli tended to cast doubt on this possibility, Experiment 2 was thought necessary to answer the question empirically.

\section{Method}

Subjects. Fifteen undergraduate psychology students, 12 women and 3 men, served as observers. None had taken part in Experiment 1. Observers ranged in age from 19 to 37 years (median $=21$ years), and all had normal or corrected-to-normal visual acuity.

Materials and apparatus. Stimuli were the same as in Experiment 1. They were projected using two Kodak Ektagraphic random-access slide projectors. One of the projectors was modified by the addition of a Potter \& Brumfield variable-timing relay (Model DKB-38-70120), which controlled exposure durations.

Procedure. Observers were seated $193 \mathrm{~cm}$ in front of a blank white wall, which served as the projection plane. Standard and comparison stimuli were presented simultaneously for $1 \mathrm{sec}$, the standard always appearing to the left of the comparison. Observers were instructed to respond "same" if the orientations of the standard and comparison figures appeared the same, or "different" if the orientations appeared to differ.

When the two figures had different orientations, the disparity could be $3^{\circ}, 6^{\circ}, 9^{\circ}, 12^{\circ}, 15^{\circ}, 18^{\circ}, 21^{\circ}$, or $24^{\circ}$. Each observer completed eight blocks of 20 trials, for a total of 160 trials. Within each block, half of the trials consisted of same orientations. Only one value of disparity was used for different orientations within a block of trials. The order of same and different trials within a block was randomized, as was the order of the eight blocks for each observer.

\section{Results}

The mean error rate and the median frequency of errors at each value of angular disparity are given in Table 1 . At and near the putative threshold for mental rotation, performance was virtually error-free.

Error rate was not highly related to trial block $(\varrho=0.38)$, indicating no pronounced effects of either practice or fatigue during these sessions.
Table 1

Discrimination of Angular Disparities in Experiment 2

\begin{tabular}{ccc}
$\begin{array}{c}\text { Comparison } \\
\text { Disparity }\end{array}$ & $\begin{array}{c}\text { Mean Error } \\
\text { Rate }\end{array}$ & $\begin{array}{c}\text { Median Error } \\
\text { Frequency }\end{array}$ \\
\hline $3^{\circ}$ & .59 & 11 \\
$6^{\circ}$ & .10 & 0 \\
$9^{\circ}$ & .04 & 0 \\
$12^{\circ}$ & .01 & 0 \\
$15^{\circ}$ & .02 & 0 \\
$18^{\circ}$ & 0 & 0 \\
$21^{\circ}$ & .01 & 0 \\
$24^{\circ}$ & .03 & 0 \\
\hline
\end{tabular}

\section{Discussion}

All disparities greater than $3^{\circ}$ can be reliably discriminated under the presentation conditions of Experiment 2 . We have confirmed this result also under different presentation conditions, using a functional measurement procedure (Rossi \& Collyer, 1981). It therefore appears that if there is a threshold at about $15^{\circ}$ in Figure 1, it reflects more than just the lower limit on an observer's ability to tell the figures apart.

\section{CONCLUSION}

There may be a threshold for mental rotation. It is likely, however, that this conjecture is far too simple. There are nonlinear mental rotation response-time functions, particularly in experiments using letter stimuli (e.g., Cooper \& Shepard, 1973), which might be interpreted using the idea of a threshold. But we would hesitate to argue strongly for the threshold and against alternative conceptions predicting a similar nonlinearity in the $a b$ sence of new data.

The slope and intercept of the large linear effect, and the form and magnitude of nonlinear effects are probably influenced by several factors. As these factors are elucidated by additional research, we shall gain a fuller picture of the flexibility and limits of mental rotation performance.

\section{REFERENCES}

Collyer, C. E. (1985). Comparing strong and weak models by fitting them to computer-generated data. Perception \& Psychophysics, 38, 476-481.

Coltheart, M. (1971). Visual feature-analyzers and aftereffects of tilt and curvature. Psychological Review, 78, 114-121.

CoOper, L. A., \& ShePard, R. N. (1973). Chronometric studies of the rotation of mental images. In W. G. Chase (Ed.), Visual information processing (pp. 75-176). New York: Academic Press.

COOPER, L. A., \& SHEPARD, R. N. (1978). Transformations on representations of objects in space. In E. C. Carterette \& M. P. Friedman (Eds.), Handbook of perception: Vol. 8. Space and object perception. New York: Academic Press.

Just, M. A., \& CARPENTER, P. A. (1976). Eye fixations and cognitive processes. Cognitive Psychology, 8, 441-480.

Rossi, J. S., \& Collyer, C. E. (1981, April). Discrimination of small angular disparities in the orientations of complex figures: $A$ functional measurement analysis. Paper presented at the 52nd annual meeting of the Eastern Psychological Association, New York.

Shepard, R. N., \& MetZleR, J. (1971). Mental rotation of threedimensional objects. Science, 171, 701-703. 